\title{
High Concentrations of Ketocarotenoids in Hepatic Mitochondria of Haemorhous mexicanus
}

ARTICLE in PHYSIOLOGICAL AND BIOCHEMICAL ZOOLOGY · MAY 2015

Impact Factor: $2.4 \cdot$ DOI: 10.1086/681992

READS

45

6 AUTHORS, INCLUDING:

Zhiyuan Ge

Auburn University

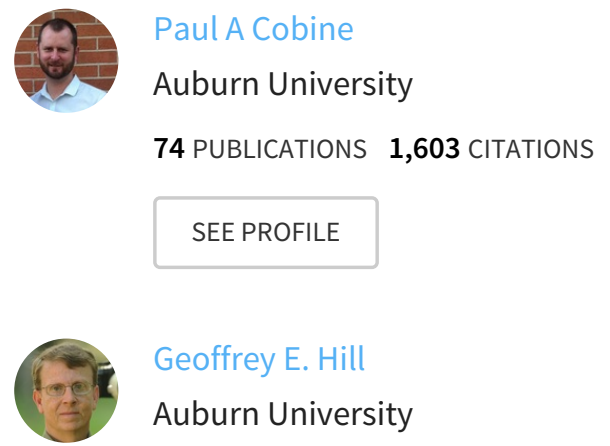

188 PUBLICATIONS 4,965 CITATIONS

234 PUBLICATIONS 9,187 CITATIONS

SEE PROFILE

SEE PROFILE 


\section{CHICAGO JOURNALS}

Division of Comparative Physiology and Biochemistry, Society for Integrative and Comparative Biology

High Concentrations of Ketocarotenoids in Hepatic Mitochondria of Haemorhous mexicanus Author(s): Zhiyuan Ge, James D. Johnson, Paul A. Cobine, Kevin J. McGraw, Rosana Garcia, and Geoffrey E. Hill

Source: Physiological and Biochemical Zoology, Vol. 88, No. 4 (July/August 2015), pp. 444-450

Published by: The University of Chicago Press. Sponsored by the Division of Comparative

Physiology and Biochemistry, Society for Integrative and Comparative Biology

Stable URL: http://www.jstor.org/stable/10.1086/681992

Accessed: 15/06/2015 09:05

Your use of the JSTOR archive indicates your acceptance of the Terms \& Conditions of Use, available at

http://www.jstor.org/page/info/about/policies/terms.jsp

JSTOR is a not-for-profit service that helps scholars, researchers, and students discover, use, and build upon a wide range of content in a trusted digital archive. We use information technology and tools to increase productivity and facilitate new forms of scholarship. For more information about JSTOR, please contact support@ jstor.org. 


\section{High Concentrations of Ketocarotenoids in Hepatic Mitochondria of Haemorhous mexicanus}

\author{
Zhiyuan $\mathrm{Ge}^{1, *}$ \\ James D. Johnson ${ }^{1}$ \\ Paul A. Cobine ${ }^{1}$ \\ Kevin J. McGraw ${ }^{2}$ \\ Rosana Garcia ${ }^{1}$ \\ Geoffrey E. Hill ${ }^{1}$ \\ ${ }^{1}$ Department of Biological Sciences, 331 Funchess Hall, \\ Auburn University, Auburn, Alabama 36849-5414; \\ ${ }^{2}$ School of Life Sciences, Arizona State University, Tempe, \\ Arizona 85287-4501
}

Accepted 4/11/2015; Electronically Published 5/7/2015

\begin{abstract}
Vertebrates cannot synthesize carotenoid pigments de novo, so to produce carotenoid-based coloration they must ingest carotenoids. Most songbirds that deposit red carotenoids in feathers, bills, eyes, or skin ingest only yellow or orange dietary pigments, which they oxidize to red pigments via a ketolation reaction. It has been hypothesized that carotenoid ketolation occurs in the liver of vertebrates, but this hypothesis remains to be confirmed. To better understand the role of hepatocytes in the production of ketolated carotenoids in songbirds, we measured the carotenoid content of subcellular components of hepatocytes from wild male house finches (Haemorhous mexicanus) that were molting red, ketocarotenoid-containing feathers (e.g., 3-hydroxy-echinenone). We homogenized freshly collected livers of house finches and isolated subcellular fractions, including mitochondria. We found the highest concentration of ketocarotenoids in the mitochondrial fraction. These observations are consistent with the hypothesis that carotenoid pigments are oxidized on or within hepatic mitochondria, esterified, and then transported to the Golgi apparatus for secretory processing.
\end{abstract}

Keywords: ketocarotenoids, mitochondria, Haemorhous mexicanus.

*Corresponding author; e-mail: zzg0008@auburn.edu.

Physiological and Biochemical Zoology 88(4):444-450. 2015. (C) 2015 by The University of Chicago. All rights reserved. 1522-2152/2015/8804-4082\$15.00. DOI: $10.1086 / 681992$

\section{Introduction}

Many species of fish, reptiles, and birds have carotenoid-based integumentary coloration. No vertebrate species can synthesize carotenoids de novo; they must ingest carotenoid pigments to use them as colorants (Fox and Vevers 1960). Once ingested, however, carotenoid pigments can be modified via enzyme-supported redox reactions. Such redox reactions can change the fundamental hue of carotenoids, including transformations of yellow dietary hydroxycarotenoids (e.g., lutein, zeaxanthin) to red ketocarotenoids (e.g., astaxanthin, canthaxanthin; Brush 1990).

Oxidation of dietary carotenoids is a particularly widespread and important source of coloration in songbirds (order Passeriformes). The diets of most songbirds contain four primary carotenoid pigments, all of which are yellow or orange: $\beta$-carotene, $\beta$-cryptoxanthin, zeaxanthin, and lutein (McGraw 2006). Some songbirds use these dietary pigments directly (Partali et al. 1987). Others oxidize lutein or zeaxanthin to produce the yellow pigments canary xanthophyll a and b (Stradi et al. 1995). Still other songbirds ketolate dietary pigments by introducing ketone groups to produce red ketocarotenoids. Ketolation of yellow dietary pigments is the primary source of red coloration in songbirds (Stradi et al. 1996, 1997; Inouye et al. 2001; fig. 1).

Extensive research has been conducted on both the function (Hill 2002, 2006) and the production (Hill and Johnson 2012; García-de Blas et al. 2015) of red carotenoid pigmentation in birds. Types and concentrations of carotenoid pigments have been characterized in internal tissues (including blood, fat, liver, and retina; Toomey and McGraw 2010) as well as in external structures with ornamental coloration (including feathers, bills, and skin; McGraw et al. 2003, 2006; McGraw and Toomey 2010). Among bird species in which carotenoids have been identified from colorful feathers and bare parts, about half display red coloration (McGraw 2006). Despite this interest in red carotenoid coloration in songbirds, the site of carotenoid metabolism remains uncertain and contentious. The liver has been proposed as the likely site of metabolism (del Val et al. 2009a, 2009b), but that hypothesis has been disputed (McGraw 2009) as universally applicable on the basis of the observations that integumentary pigments are absent from liver and plasma in some species (García-de Blas et al. 2015; McGraw 2004). More recently, it was proposed that hepatic carotenoid metabolism should occur within or in close association with mitochondria because (1) evolutionary relationships exist between carotenoids and quinones (Johnson and Hill 2013); (2) oxidation potential is adequate at the inner mitochondrial member (Johnson and Hill 


\section{Dietary Carotenoids}<smiles>CC1=C(/C=C/C(C)=C/C=C/C(C)=C/C=C/C(C)=C/C=C/C=C(C)/C=C/C=C(C)/C=C/C=C(C)/C=C/C=C(C)/C=C/C2=C(C)CCCC2(C)C)C(C)(C)CCC1</smiles>

\section{Red Ketolated Carotenoids}<smiles>C/C=C(/C=C/C(C)=C/C=C/C(C)=C/C=C/C=C/C(C)=C/C=C/C1=C(C)CCCC1(C)C)C1(C)CCCC(=O)C1</smiles>

3-hydroxy-echinenone<smiles>CC1=C(/C=C/C(C)=C/C=C/C=C(C)/C=C/C=C(C)/C=C/C=C/C(C)=C/C=C/C2=C(C)C(C)(C)CCC2C)C(C)(C)C[C@@H](O)C1=O</smiles>

Astaxanthin<smiles>CC1=C(/C=C/C(C)=C/C=C/C=C(C)/C=C/C=C/C=C(C)/C=C/C=C(C)/C=C/C2=C(C)C(=O)[C@@H](O)CC2(C)C)C(C)(C)C[C@H](O)C1=O</smiles><smiles>C/C=C/C=C(C)/C=C/C=C/C=C(C)/C=C/C=C(C)/C=C/C1=C(C)C(=O)CCC1(C)C</smiles>

Figure 1. Chemical structure of the primary carotenoids that are in the diets of songbirds and that are metabolically derived through ketolation reactions. Portions in red indicate the positions of ketone groups that are the product of biochemical modification within birds.

2013); (3) the mitochondria are closely linked to mechanisms for transport and packaging of lipids and lipid-like molecules, including carotenoids (Hill and Johnson 2012); (4) there are plausible oxidation sites, such as complex III, that could serve as a location for carotenoid oxidation (Johnson and Hill 2013); and (5) carotenoids are known to be transported to the mitochondria in some bird species (Mayne and Parker 1986).

Here, we studied pigment accumulation in the livers of male house finches (Haemorhous mexicanus) that were undergoing molt and hence synthesizing ketocarotenoids from dietary carotenoids. Our goal was to describe the specific subcellular locations of carotenoids in a putative site for production of ornamental red pigments. Male house finches have extensive carotenoid plumage coloration that varies from red to yellow and that is developed in a relatively short window of time in the late summer during prebasic molt (Hill 2002). On the basis of likely precursor-product relationships, it has been argued that house finches derive their red coloration by oxidizing primarily dietary $\beta$-cryptoxanthin into primarily the red ketocarotenoid 3-hydroxy-echinenone
(Inouye et al. 2001; McGraw et al. 2006). We collected wild molting male house finches and analyzed the carotenoid content of cellular fractions of homogenized liver to test a key assumption of the hypothesis that carotenoid pigments used for ornamental feather coloration are oxidized in hepatic mitochondria. Within this context, high concentration of ketocarotenoids would be expected inside the mitochondria, while the level of precursor $\beta$-cryptoxanthin should be much lower.

\section{Material and Methods}

Collection of Liver Samples

In August 2014, four hatching-year molting house finches were captured at a feeding station in Lee County, Alabama $\left(32^{\circ} 35^{\prime} 46.480^{\prime \prime} \mathrm{N},-85^{\circ} 29^{\prime} 29.270^{\prime \prime} \mathrm{W}\right)$. Males were killed by asphyxiation in $\mathrm{CO}_{2}$ chamber, following protocols approved by the Institutional Animal Care and Use Committee at Auburn University. The whole liver of each male was removed within 1 min of euthanasia and, within 20 s, chilled on ice. Livers 
and extracts were kept on ice throughout the process of mitochondria isolation.

\section{Mitochondrial Preparation}

Mitochondria were prepared by differential centrifugation, using the protocol of Trounce et al. (1996) modified by Ingraham and Pinkert (2003). Within $20 \mathrm{~s}$ of dissection from carcasses $(n=4)$, livers were rinsed and minced in mitochondria isolation buffer. During the entire procedure, each bird's liver was processed separately, and all liver mitochondria preparations were kept at $4^{\circ} \mathrm{C}$. Minced livers were then homogenized with a $2-\mathrm{mL}$ glass Teflon homogenizer at $300 \mathrm{mg}$ tissue per milliliter of buffer. The crude homogenate was centrifuged twice at $750 \mathrm{~g}$ for $10 \mathrm{~min}$. Remaining pellets were collected and resuspended in $1 \mathrm{~mL}$ SMEE as fraction 1 . The $750 \mathrm{~g}$ supernatant was centrifuged for $15 \mathrm{~min}$ at $9,800 \mathrm{~g}$, and the resulting supernatant was collected carefully as fraction 2 . The pellet was resuspended slowly by drop-wise addition of SMEE. This suspension was then centrifuged for $10 \mathrm{~min}$ at 9,800 $g$ again, and supernatant for this spin was collected as fraction 3. The final purified mitochondria pellets were resuspended in $1 \mathrm{~mL}$ SMEE (fraction 4 ) and stored at $-80^{\circ} \mathrm{C}$.
Immunocytochemistry: Western Blot Analysis

We used marker proteins to identify fractions isolated by centrifugation containing mitochondria. Total protein concentration for the various fractions was determined using Bradford assay (Bradford 1976), with fat-free BSA as the standard. A Shimadzu UV-2450 spectrophotometer (Chiyoda-ku, Tokyo) was used to measure absorbance of the Bradford reagent at $595 \mathrm{~nm}$. We made a standard curve of protein concentration that we used to estimate the protein content of isolated fractions.

Immunoblots of putative mitochondria pellets were performed using rabbit polyclonal antibody adenine nucleotide translocator proteins $1,2,3$, and 4 (ANT1/2/3/4, Santa Cruz Biotechnology), which is a multipass membrane protein of the mitochondrial inner membrane that can be used to quantify mitochondria (Olvera-Sanchez et al. 2011). Isolated mitochondria extracts were suspended in $1 \times$ phosphate-buffered saline (PBS) solution and incubated in boiling water for $5 \mathrm{~min}$ to break the mitochondria membrane and denature the proteins released. Proteins $(28.5 \mu \mathrm{g} /$ well $)$ were separated by SDS-PAGE in $10 \%$ gels at $200 \mathrm{~V}$ for $40 \mathrm{~min}$ and transferred to polyvinylidene fluoride (PVDF) membranes via a semidry electrophoretic transfer
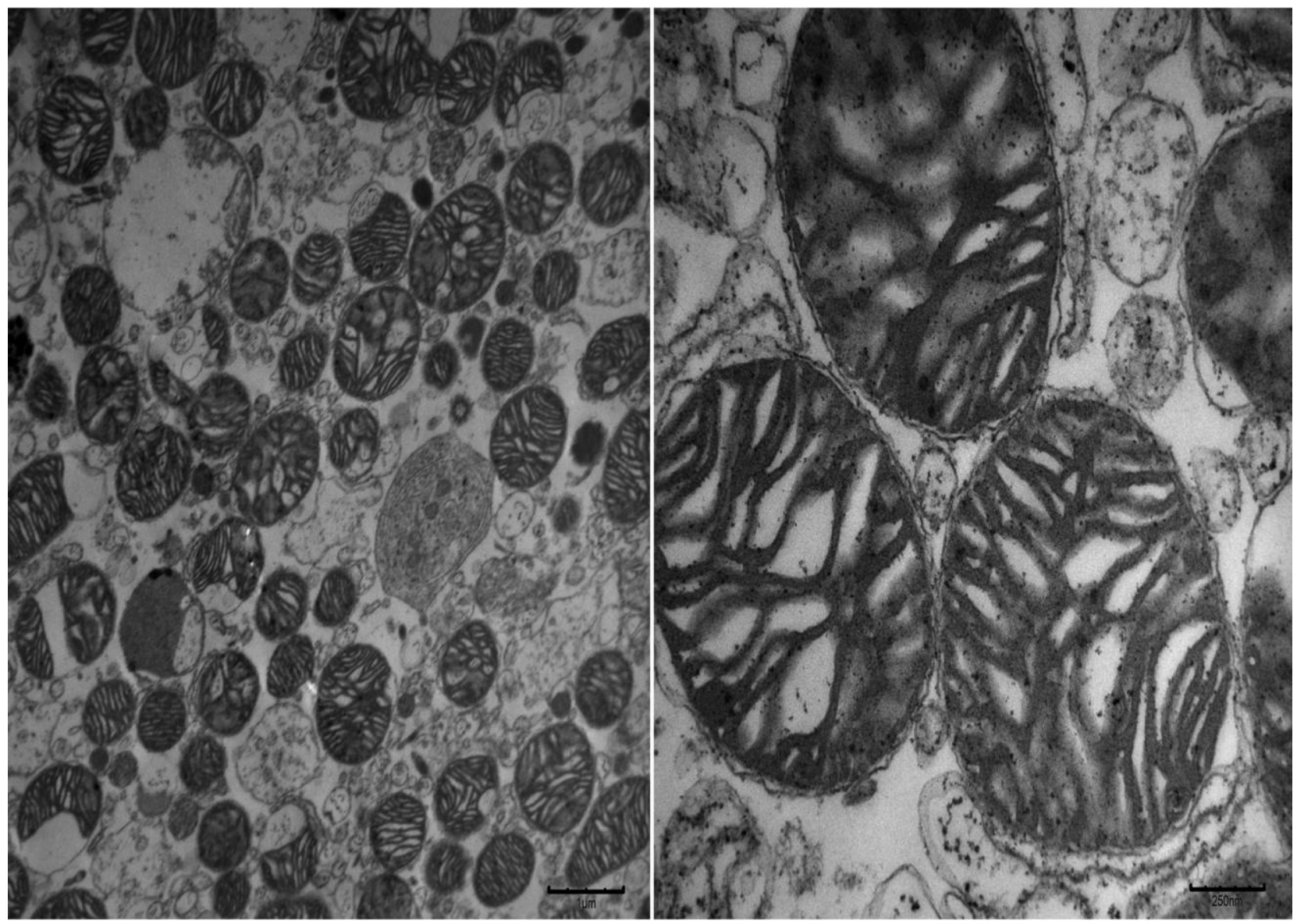

Figure 2. Images of fraction 4 in the mitochondrial isolation series used in this study made with a TEM at a magnification of $\times 12,500$ (left) and $\times 50,000($ right $)$. Mitochondria appear unbroken and are present in high density. 


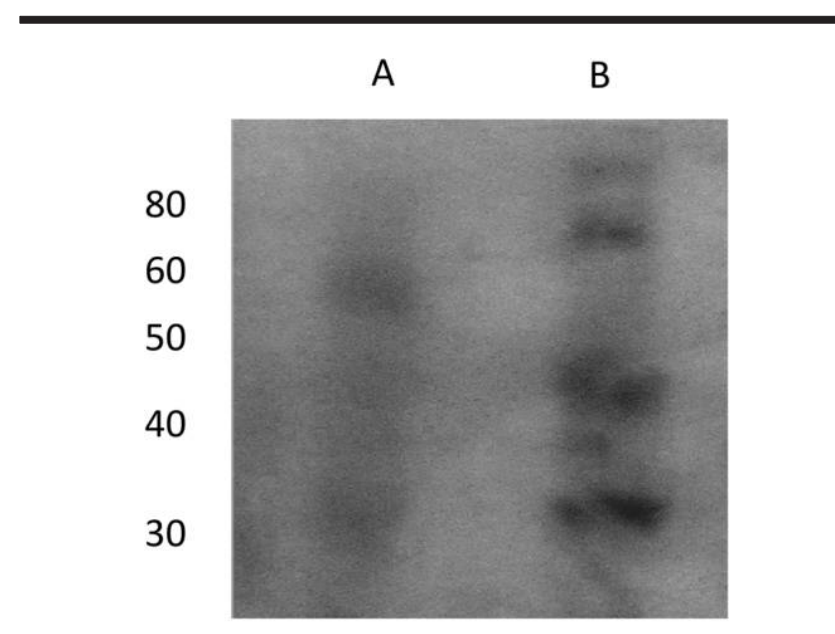

Anti- ANT1/2/3/4

Figure 3. Immunoblot analysis of the mitochondrial protein ANT1/2/ $3 / 4$ in fractions from mitochondrial isolation protocol. Lane A is the supernatant from the initial high speed spin (fraction 2), and lane B is the enriched mitochondrial pellet (fraction 4). Equal protein loading as determined by Bradford assay. The darker staining in lane B indicates a higher concentration of mitochondria in fraction 4.

cell at $20 \mathrm{~V}$ for $30 \mathrm{~min}$. Membranes were washed three times for 5 min each with PBS containing 0.05\% Tween-20 (wash buffer), followed by blocking with $5 \%$ nonfat powdered milk in washing buffer for $90 \mathrm{~min}$. Primary polyclonal antibody for ANT1/2/3/4 (Santa Cruz Biotechnology) was diluted by 1/1,000 in wash buffer, respectively. Membranes were incubated with primary antibody at room temperature overnight $(12 \mathrm{~h})$, followed by two 5 -min washes. The secondary antibody (goat antirabbit IgG [heavy and light chain] antibody) was diluted to $1 / 5,000$ in wash buffer before being added to membranes for $40 \mathrm{~min}$ incubation. Membranes were then washed three times before visualization onto photographic x-ray film with Amersham ECL select (GE Healthcare, Pittsburgh), according to manufacturers' protocols.

\section{Carotenoid Analysis}

Methods for carotenoid extraction and high-performance liquid chromatography (HPLC) analyses generally follow those of McGraw and Toomey (2010). We sequentially extracted carotenoids from $10 \mathrm{~mL}$ of each thawed liver fraction using ethanol and then $1: 1(\mathrm{v} / \mathrm{v})$ hexane:methyl tert butyl ether, vortexing for $5 \mathrm{~s}$ after adding each solvent. We centrifuged the tubes, transferred the colored supernatant to a fresh tube, and evaporated the solution to dryness under a stream of nitrogen. We resuspended residue in $200 \mu \mathrm{L}$ HPLC mobile phase (42:42:16 methanol:acetonitrile:dichloromethane, $\mathrm{v} / \mathrm{v} / \mathrm{v})$ and injected $50 \mu \mathrm{L}$ into a Waters Alliance HPLC instrument (Waters, Milford, MA) equipped with a reverse-phase C-30 YMC carotenoid column (YMC, Kyoto) and a column heater set to $30^{\circ} \mathrm{C}$. The column was pretreated with $1 \%$ orthophosphoric acid to permit recovery of ketocarotenoids (Toomey and
McGraw 2007). Runs consisted of isocratic elution in the mobile phase for $11 \mathrm{~min}$, followed by a linear gradient that finished at 42:23:35 methanol:acetonitrile: dichloromethane at $21 \mathrm{~min}$; these conditions were held until $25 \mathrm{~min}$, at which point the run returned to initial conditions until $29.5 \mathrm{~min}$. Carotenoids were identified by comparison to standards and quantified using external standard curves. We detected lutein, $\beta$-carotene, and 3-hydroxy-echinenone in several forms (cis isomers plus in pure and esterified form), and all data are presented in carotenoids/10 mg protein (Palczewski et al. 2014).

\section{Results}

\section{Identification of Isolated Liver Fractions}

On the basis of validations in previous studies of the standard protocols that we employed (Johnson et al. 1967; Prudent et al. 2013), we classified fraction 1 that was deep brownish redthe same color as homogenized whole liver-to contain mostly tissue debris and blood. The soluble fraction of the second centrifugation step (fraction 2) should contain microsomes, fat, soluble debris, broken mitochondria membrane, and other organelle debris. Fraction 2 had a slight orange color. Fraction 3, a second supernatant, is proposed to be the suspension of similar cellular fraction residues from fraction 2 and was pale in color with the lowest protein concentration among all fractions, suggesting that most microsomal and cytosolic components were removed after the first high-speed spin. Finally, we took fraction 4 to be the crude mitochondria pellet. It was orange-red in color. Support for fraction 4 being a mitochondrial pellet came from direct observation of the fraction using TEM. The TEM image shows dense and unbroken mitochondria in fraction 4 (fig. 2). We also used polyclonal antibody for ANT1/2/3/4, which is a mitochondrial marker to test fractions for mitochondrial content. ANT1/2/3/4 increased in concentration through the extraction steps, with the highest concentration in fraction 4 , the mitochondria pellet (fig. 3). Few total proteins were detected in fraction 3, so it was not included in western blot analysis.

\section{Carotenoid Concentration}

Carotenoids were detected in three fractions, but the highest concentration was in fraction 4 , the crude mitochondrial pellet (fig. 4). Approximately $85 \%$ of the total carotenoids as well as $67 \%$ of the total ketocarotenoids were found in the crude mitochondrial pellet. Ketocarotenoids were nearly twice as abundant in the crude mitochondrial fraction, as were other carotenoids, suggesting that ketocarotenoids were located either within hepatic mitochondria or closely associated with them.

\section{Discussion}

Red pigmentation of the feathers and bare parts of birds has been the focus of intensive research by behavioral and evolutionary biologists interested in understanding the function and evolution of ornamentation (Hill 2002; Hill and McGraw 2006). The physiological mechanisms that underlie the production of 


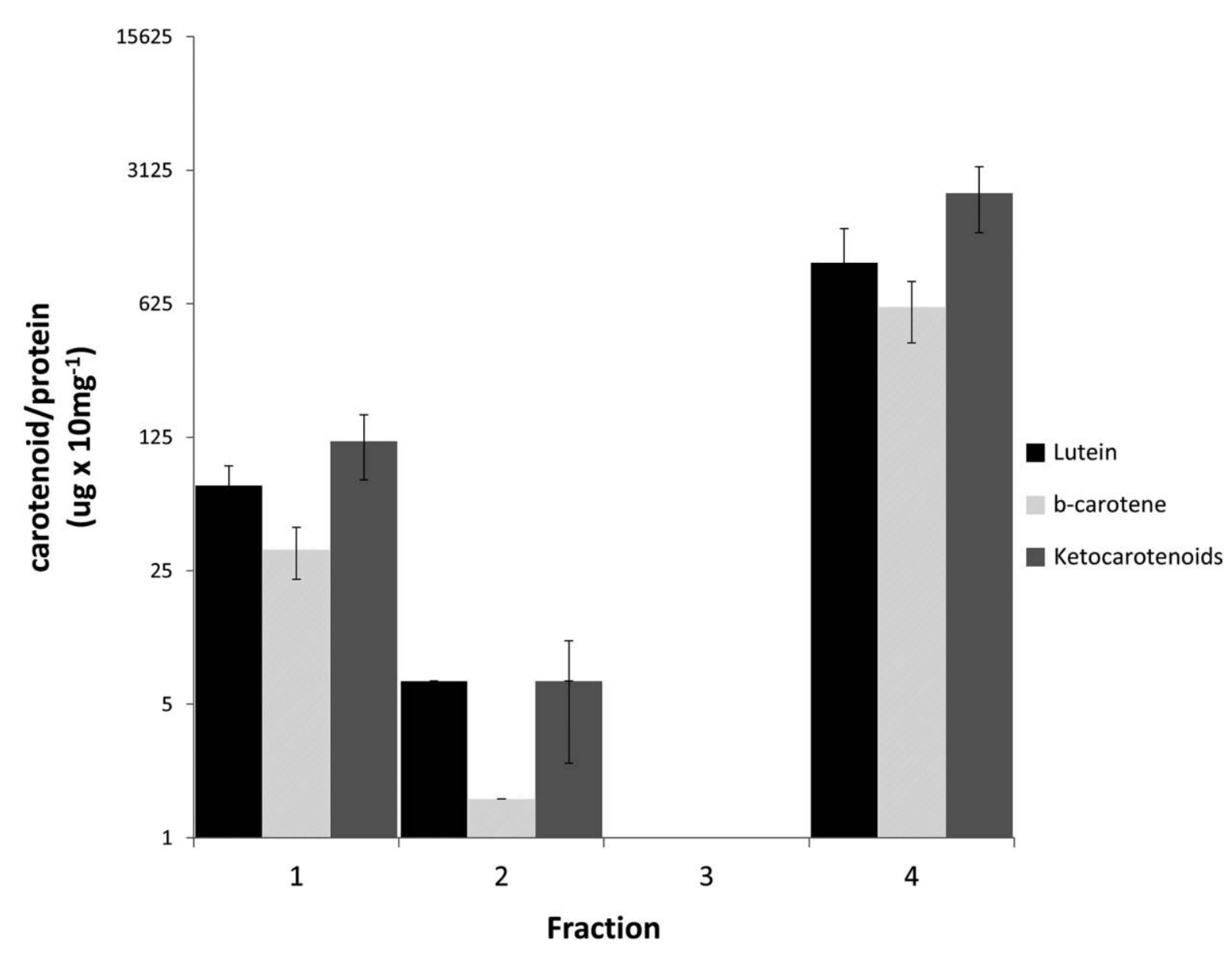

Figure 4. Concentration of carotenoids in four fractions from liver tissue of molting male house finches. Carotenoids were found in three fractions. The crude mitochondria pellets (fraction 4) had the highest concentration of ketocarotenoids. Data represent means \pm SD of four birds/data point.

ornamental coloration are central to hypotheses related to the signal content of red coloration (Hill 2011; Hill and Johnson 2012; García-de Blas et al. 2015). Yet, key aspects of the production of red coloration remain unstudied. Here we made the first attempt to determine the carotenoid content of subcellular fractions of hepatic tissue and determine the site of accumulation of metabolically produced ketocarotenoids in a songbird that uses these pigments for integumentary coloration. We detected high concentrations of carotenoids in the mitochondria of hepatocytes, which turned out to be 15 times higher (carotenoids/10 mg protein) than in the mitochondria of spleen and brain of these same birds and 10 times higher than in the mitochondria of heart (Z. Ge and G. E. Hill, unpublished data). These observations suggest that the liver is a major site of ketolation or storage of the red carotenoid pigments used by songbirds to color feathers. In other orders of birds and especially for carotenoid coloration of bare parts, ketolation may occur at sites other than the liver, such as the integument (García-de Blas et al. 2015). More comparative data are certainly needed.

Our data suggest that the subcellular location of carotenoid ketolation is within mitochondria, on the outer membrane of mitochondria, or in close association with Golgi apparatus- linked mitochondria-associated endoplasmic reticulum membranes. As we proceeded toward more purified hepatic mitochondria through rounds of centrifugation, ketocarotenoid concentrations in the mitochondrial layer increased, such that the enriched mitochondrial fraction had the highest levels of ketocarotenoids. Microscopic examination of this fraction also showed a high density of unbroken mitochondria. Because of the limits of the mitochondria isolation technique, there was undoubtedly loss and damage of intact mitochondria in each round of centrifugation, leading to carotenoids leaking from ruptured mitochondrial membranes. The first high-speed supernatant (fraction 2) would be expected to have the highest concentration of carotenoids released from the breakage of mitochondria because of the attraction of carotenoids to the high fat content of debris, and that is what we observed.

High concentration of ketocarotenoids in mitochondria of hepatocytes is consistent with a key prediction of the hypothesis that oxidation of dietary carotenoids occurs in mitochondria of liver cells (Hill and Johnson 2012; Johnson and Hill 2012) and that the liver is a primary site of red carotenoid ketolation in red cardueline finches (del Val et al. 2009a, 2009b). This conclusion, however, rests on the assumption that a high 
concentration of ketolated carotenoids is indicative of the sit $\rightarrow$ Brush A.H. 1990. Metabolism of carotenoid pigments in birds. where the ketolation occurred. In our study, low levels of $\beta$ FASEB J 4:2969-2977.

cryptoxanthin were detected in crude mitochondria pellets, sug $\rightarrow$ del Val E., J.C. Senar, J. Garrido-Fernandez, M. Jaren, A. Borras, gesting the presence of the substrate for ketolation; almost no $\beta$-cryptoxanthin was found in other fractions (data not presented). Low levels of $\beta$-cryptoxanthin have also been detectec $\rightarrow$ in plasma in a recent analysis of plasma of molting house finches from Arizona (Giraudeau and McGraw 2014), suggesting the possibility of rapid conversion from $\beta$-cryptoxanthin to 3 -hydroxy echinenone. More data are needed to demonstrate that hepatic mitochondria are the primary site of metabolism of the red pigments used to color the feathers of songbirds. Even if technically difficult, an informative approach would be to use radiolabeled carotenoids or stable carotenoid tracers to track production path ways for carotenoids (Schiedt 1998).

Our observations of high concentrations of carotenoids within or in intimate association with the mitochondria of the hepatic cells of molting hatching-year male house finches hav important implications for the cellular mechanisms that regulate the production of ornamental plumage coloration. Ornament elaboration is a function of the redness of plumag $\rightarrow$ display in the house finch and other birds (Hill 1996), and plumage redness is a function of the ratio of ketocarotenoids versus xanthophylls deposited in feathers (Inouye et al. 2001; McGraw et al. 2006). If carotenoid ketolation occurs within or on the outer surface of mitochondria, then carotenoid oxidation will likely be linked to core electron transporting events, including cytochrome b5 and vitamin $\mathrm{C}$ reduction on the outer mitochondrial membrane and oxidative phosphorylation or $\rightarrow$ the inner mitochondria membrane. The efficiency of the ketolation process is likely to reflect core electron transport effi $\rightarrow$ ciency, and this should be reflected in plumage coloration (Hill 2011; Johnson and Hill 2013). Females can potentially asses: $\rightarrow$ the efficiency of cellular respiration of prospective mates by assessing plumage redness (Hill 2014). This hypothesis could be tested directly by relating the redness of growing feathers tc respiratory efficiency. In future studies, it would be informative to obtain more highly purified hepatic mitochondria from density centrifugation to better address the direct correlation beJ. Cabrera, and J.J. Negro. 2009a. Hepatic conversion of red carotenoids in passerine birds. Naturwissenschaften 96:989-991. 2009b. The liver but not the skin is the site for conversion of a red carotenoid in a passerine bird. Naturwissenschaften 96:797-801.

$\rightarrow$ Diekert K., A. de Kroon, G. Kispal, and R. Lill. 2001. Isolation and subfractionation of mitochondria from the yeast Saccharomyces cerevisiae. Methods Cell Biol 65:37-51.

Fox H.M. and G. Vevers. 1960. The nature of animal colors. Macmillan, New York.

$\rightarrow$ García-de Blas E., R. Mateo, and C. Alonso-Alvarez. 2015. Accumulation of dietary carotenoids, retinoids and tocopherol in the internal tissues of a bird: a hypothesis for the cost of producing colored ornaments. Oecologia 17:259-271.

Giraudeau M. and K.J. McGraw. 2014. Physiological correlates of urbanization in a desert songbird. Integr Comp Biol 54: 622-632.

$\rightarrow$ Hill G.E. 1996. Redness as a measure of the production cost of ornamental coloration. Ethol Ecol Evol 8:157-175.

2002. A red bird in a brown bag: the function and evolution of ornamental plumage coloration in the house finch. Oxford University Press, New York.

2006. Female choice for ornamental coloration. In G.E. Hill and K.J. McGraw, eds. Bird coloration. Vol. 2. Function and evolution. Harvard University Press, Cambridge, MA.

2011. Condition-dependent traits as signals of the functionality of vital cellular processes. Ecol Lett 14:625-634.

2014. Cellular respiration: the nexus of stress, condition, and ornamentation. Integr Comp Biol 54:645-657.

Hill G.E. and J.D. Johnson. 2012. The vitamin A-redox hypothesis: a biochemical basis for honest signaling via carotenoid pigmentation. American Naturalist 180:E127-E150.

$\rightarrow$ Hoppel C.L., J. Kerner, P. Turkaly, J. Turkaly, and B. Tandler. 1998. The malonyl-CoA-sensitive form of carnitine palmitoyltransferase is not localized exclusively in the outer membrane of rat liver mitochondria. J Biol Chem 273:23495-23503. tween carotenoids ketolation and functional status of mito $\rightarrow$ Ingraham C.A. and C.A. Pinkert. 2003. Developmental fate chondria of avian hepatic cells.

\section{Acknowledgments} of mitochondria microinjected into murine zygotes. Mitochondrion 3:39-46. doi:10.1016/S1567-7249(03)00075-8.

We were supported by National Science Foundation grant IOS200272 during the preparation of this manuscript. Wendy Hood, Haruka Wada, and the Hill/Hood/Wada Lab groups commented on an earlier version of the manuscript.

$\rightarrow$ Inouye C.Y., G.E. Hill, R. Montgomerie and R.D. Stradi. 2001. Carotenoid pigments in male house finch plumage in relation to age, subspecies, and ornamental coloration. Auk 118: 900-915.

$\rightarrow$ Johnson D. and L. Henry. 1967. Isolation of liver or kidney mitochondria. Methods Enzymol 10:94-96.

\section{Literature Cited}

$\rightarrow$ Johnson J.D. and G.E. Hill. 2013. Is carotenoid ornamentation linked to the inner mitochondria membrane potential? a hypothesis for the maintenance of signal honesty. Biochimie 95:436-444.

$\rightarrow$ Bradford M.M. 1976. A rapid and sensitive method for th $\rightarrow$ Mayne S.T. and R.S. Parker. 1986. Subcellular distribution of quantitation of microgram quantities of protein utilizing dietary $\beta$-carotene in chick liver. Lipids 21:164-169. the principles of protein-dye binding. Anal Biochem $72 \rightarrow$ McGraw K.J. 2004. Colorful songbirds metabolize carotenoids $248-254$. at the integument. J Avian Biol 35:471-476. 
2006. Mechanics of carotenoid coloration. In G.E. Hill and K.J. McGraw, eds. Bird coloration. Vol. 1. Measurements and mechanisms. Harvard University Press, Cam bridge, MA.

$\rightarrow$ McGraw K.J., A.J. Gregory, R.S. Parker, and E. Adkins-Regan. 2003. Diet, plasma carotenoids, and sexual coloration in the zebra finch (Taeniopygia guttata). Auk 120:400-410.

$\rightarrow-$ 2009. Identifying anatomical sites of carotenoid me tabolism in birds. Naturwissenschaften 96:987-988.

$\rightarrow$ McGraw K.J., P.M. Nolan, and O.L. Crino. 2006. Carotenoid accumulation strategies for becoming a colourful house finch: analyses of plasma and liver pigments in wild moulting birds Funct Ecol 20:678-688.

$\rightarrow$ McGraw K.J. and M.B. Toomey. 2010. Carotenoid accumulation in the tissues of zebra finches: predictors of integumentary pigmentation and implications for carotenoid allo cation strategies. Physiol Biochem Zool 83:97-109.

$\rightarrow$ Olvera-Sanchez S., M.T. Espinosa-Garcia, J. Monreal, O. Flores-Herrera, and F. Martinez. 2011. Mitochondrial heat shock protein participates in placental steroidogenesis. $\mathrm{Pla}$ centa 32:222-229.

$\rightarrow$ Palczewski G., J. Amengual, C.L. Hoppel, and J. von Lintig. 2014. Evidence for compartmentalization of mammalian carotenoid metabolism. FASEB J 28:4457-4469.

Partali V., S. Liaaen-Jensen, T. Slagsvold, and J.T. Lifjeld. 1987. Carotentoids in food chain studies. II. The food chain of Parus spp. monitored by carotenoid analysis. Comp Biochem Physiol 87B:885-888.

Prudent J., N. Popgeorgeiv, B. Bonneau, and G. Gillet. 2013 Subcellular fractionation of zebrafish embryos and mitochondrial calcium uptake exchange. Protocol Exchange, doi: 10.1038/protex.2013.073.
Schiedt K. 1998. Absorption and metabolism of carotenoids in birds, fish and crustaceans. Carotenoids 3:285-358.

Schwartz M.K. 1973. Enzymes in cancer. Clin Chem 19:10-22.

$\rightarrow$ Stradi R., G. Celentano, M. Boles, and F. Mercato. 1997. Carotenoids in bird plumage: the pattern in a series of redpigmented Carduelinae. Comp Biochem Physiol 117B:8591.

$\rightarrow$ Stradi R., G. Celentano, E. Rossi, G. Rovati, and M. Pastore. 1995. Carotenoids in bird plumage. I. The carotenoid pattern in a series of Palearctic Carduelinae. Comp Biochem Physiol 110B:131-143.

$\rightarrow$ Stradi R., E. Rossi, G. Celentano, and B. Bellardi. 1996. Carotenoids in bird plumage: the pattern in three Loxia species and in Pinicola enucleator. Comp Biochem Physiol 113B:427-432.

Toomey M.B. and K.J. McGraw. 2007. Modified saponification and HPLC methods for analyzing carotenoids from the retina of quail: implications for its use as a nonprimate model species. Invest Ophthalmol Vis Sci 48:3976-3982.

$\rightarrow$ - 2010. The effects of dietary carotenoid intake on carotenoid accumulation in the retina of a wild bird, the house finch (Carpodacus mexicanus). Arch Biochem Biophys 504:161-168.

$\rightarrow$ Tran K., G. Thorne-Tjomsland, C.J. DeLong, Z. Cui, J. Shan, L. Burton, J.C. Jamieson, and Z.M. Yao. 2002. Intracellular assembly of very low density lipoproteins containing apolipoprotein B100 in rat hepatoma McA-RH7777 cells. J Biol Chem 277:31187-31200.

Trounce I.A., Y.L. Kim, A.S. Jun, and D.C. Wallace. 1996. Assessment of mitochondrial oxidative phosphorylation in patient muscle biopsies, lymphoblasts, and transmitochondrial cell lines. Methods Enzymol 264:484-509. 